SYNTHETIC COMMUNICATIONS, 32(3), 457-465 (2002)

\title{
A SOLVENT-FREE AND FORMALIN-FREE ESCHWEILER-CLARKE METHYLATION FOR AMINES
}

\author{
Thomas Rosenau, ${ }^{1}$ Antje Potthast, ${ }^{1}$ Jürgen Röhrling, ${ }^{1}$ \\ Andreas Hofinger, ${ }^{1}$ Herbert Sixta, ${ }^{2}$ and Paul Kosma ${ }^{1}$,* \\ ${ }^{1}$ Institute of Chemistry, Christian-Doppler-Laboratory, \\ University of Agricultural Sciences, Vienna, \\ Muthgasse 18, A - 1190 Vienna, Austria \\ ${ }^{2}$ R\&D Lenzing AG, A - 4860 Lenzing, Austria
}

\begin{abstract}
Primary and secondary amines are $N$-methylated by a mixture of paraformaldehyde and oxalic acid dihydrate in good to excellent yields. The reaction proceeds without involvement of organic solvents and toxic formalin. Reaction temperatures of $100^{\circ} \mathrm{C}$ are required for the decomposition of oxalic acid into the intermediate formic acid which acts as the actual reductant. The reaction conditions have been optimized, and the mechanism has been elucidated by means of deuteration experiments.
\end{abstract}

*Corresponding author. Fax: + 431360066059 ; E-mail: pkosma@edv2.boku.ac.at

457

www.dekker.com 


\section{INTRODUCTION}

$N$-Methylation of nucleophilic nitrogen in amines is a common procedure in both lab-scale and bulk-scale organic synthesis. The use of alkylating agents, such as methyl iodide or methyl tosylate, has often the disadvantage of competing quaternization of the amine, so that the Eschweiler-Clarke methylation procedure ${ }^{1,2}$ is applied instead. This approach, in principle a variant of the well-known Leuckart-Wallach reaction, ${ }^{3,4}$ uses aqueous formaldehyde solution (formalin) together with concentrated formic acid. The $N$-methylation is effected in two steps: formation of $N$-(methylene)iminium cation intermediates via the $N$-hydroxymethyl derivatives, and subsequent reduction by formic acid, which is oxidized to carbon dioxide in turn.

Practical application of the Eschweiler-Clarke methylation follows a well-established protocol that ensures its wide applicability. In this paper, a $N$-methylation approach is presented, which differs from the conventional practice and includes some distinct improvements over the existing methodology in terms of simplicity of the work-up and use of hazardous chemicals: The use of toxic formaldehyde and organic solvents is avoided, and rather time-consuming and tedious purification steps, such as column chromatography purification or steam distillation, which were hitherto required in most cases, are not required.

\section{RESULTS AND DISCUSSION}

During our investigations on deoxygenative demethylations of $N$-methylmorpholine- $N$-oxide (1) ${ }^{5}$ we observed that $N$-methylmorpholine (2) was found in addition to the expected products morpholine (3) and formaldehyde (4), if traces of oxalic acid (5) were present. Increasing the amount of oxalic acid in the reaction mixture shifted the product distribution towards higher yields of $N$-methylmorpholine. This was evidently due to a reduction of intermediate $N$-(methylene)morpholinium cations (6), so that the formation mechanism resembled a Leuckart-Wallach reaction. This result was intriguing insofar as oxalic acid is well-known as a reductant, but not as a cryptobase or hydride transfer reagent ${ }^{6}$ as needed for these reductions to proceed.

Further experiments revealed that oxalic acid is indeed able to act as a reductant in mixtures of primary amines or secondary amines and formalin, albeit at elevated temperatures around $100^{\circ} \mathrm{C}$. The resulting $N$-methylamines or $N, N$-dimethylamines, respectively, were obtained in rather low yields below $10 \%$, however. The use of solid paraformaldehyde (4a) instead 
of formalin provided a distinct improvement. The yields were significantly increased, so that further optimization towards synthetic usability seemed to be justified.

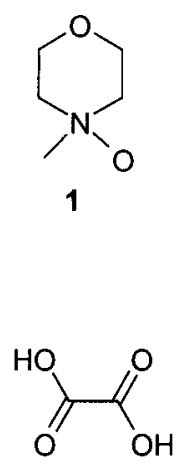

5<smiles>[2H]N1CCOCC1</smiles>

$2 \mathrm{R}=\mathrm{CH}_{3}$

2a $\mathrm{R}=\mathrm{CH}_{2} \mathrm{D}$

2b R $=\mathrm{CHD}_{2}$<smiles>O=C(O)C(=O)O</smiles>

$5 a$<smiles>C1COCCN1</smiles>

3

.

4<smiles>C=O</smiles>

4<smiles>[CH2-][N+]1=CCOCC1</smiles>

6

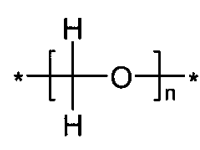

$4 a$<smiles>O=CN1CCOCC1</smiles>

7

Finally, a clean methylation of secondary amines in yields above $90 \%$ was achieved with an equimolar ratio of secondary amine and paraformaldehyde along with the fivefold molar amount of oxalic acid dihydrate (5a) within one hour. The same good result was obtained for the bismethylation of primary amines, now employing amine, $4 \mathbf{a}$ and $\mathbf{5 a}$ in the molar ratio $1: 2: 10$. Carried out at $100-120^{\circ} \mathrm{C}$, the reaction is a solvent-free process that proceeds in the molten state. The formaldehyde required is generated in situ from paraformaldehyde by thermal or acid-catalyzed decomposition.

The primary product obtained is the corresponding ammonium oxalate. The free base can be conveniently obtained by treatment of the product mixture with aqueous calcium hydroxide followed by extraction, or by treatment with a suspension of calcium oxide in ethyl acetate or ethanol. One advantage of the presented $N$-methylation procedure is the purity of the amine obtained which is indicative of an almost complete absence of side reactions. No quaternization of the amines was observed.

For optimization of the reaction conditions the system morpholine (3) $/ \mathrm{N}$ methylmorpholine (2) was used. In preliminary experiments it was established that an equimolar ratio between amine and paraformaldehyde (4a) was required to avoid side reactions. Already a small excess of $10 \%$ 4a caused the formation of a complex product mixture with severe discoloration of the reaction mixture 
due to condensation processes. The molar ratio of amine and 4a was consequently kept constant at $1 / 1$ throughout the experiments.

The stoichiometric amount of oxalic acid (5) employed proved to be a crucial issue in terms of conversion of the starting material and purity of the methylated product. At a ratio below 1, relative to the amine 3 and paraformaldehyde (4a), mainly unreacted starting amine besides small amounts of the methylated amine were found. Formation of byproducts was not observed. At increasing ratios up to 5, the product yield increased accordingly up to about $80 \%$. Interestingly, small amounts $(<10 \%)$ of $N$-formylmorpholine (7) were found, traces of several unidentified reaction products, in sum less than $3 \%$, were also detected. The highest yield of byproduct 7 was obtained at a molar ratio $\mathbf{3} / \mathbf{4 a} / \mathbf{5}=1 / 1 / 3$. The occurrence of such formylated compounds is a strong indication of the intermediate occurrence of formic acid from the decomposition of oxalic acid. This is in complete agreement with experimental and theoretical studies on the thermal decomposition of oxalic acid. ${ }^{7,8}$

Experimental studies have firmly established that the major decomposition products of pure oxalic acid over the temperature range $400-430 \mathrm{~K}$ are equimolar quantities of formic acid and carbon dioxide ${ }^{7}$ if other reaction routes, such as metal ion catalysis, catalysis by strong acids or decomposition at the walls of the reaction vessel, which lead to formation of equimolar amounts of $\mathrm{CO}_{2}, \mathrm{CO}$ and water, can be excluded. High-level quantum mechanical calculations ${ }^{8}$ indicate that this reaction is a bimolecular process: in a first step oxalic acid fragments into carbon dioxide and dihydroxycarbene. In the rate determining step dihydroxycarbene reacts with oxalic acid or water to be rearranged to formic acid. The unimolecular channel to produce $\mathrm{CO}_{2}, \mathrm{CO}$ and water becomes more prominent only with increasing temperature. At higher molar ratios, above 5, formation of both $\mathbf{7}$ and trace byproducts was completely suppressed, but up to an eightfold excess of $\mathbf{5}$ conversion of 3 to the product was incomplete. A ratio of $1 / 1 / 10$ reliably allowed for a complete consumption of starting amine and neat $N$-methylation in quantitative or nearly-quantitative yields.

As oxalic acid (5) does not solely act as the reductant, but also as the hydrogen donor in the system, oxalic acid dihydrate (5a), possessing hydrate water as an additional proton source, was employed instead. With $\mathbf{5 a}$ as the reductant, the amount of oxalic acid required could be reduced to 50 percent without impairing yield or product purity. Hence, the optimum molar ratio amine/paraformaldehyde (4a)/oxalic acid dihydrate (5a) employed was $1 / 1 / 5$. This starting material composition was used throughout the methylation experiments on different amines and for all further mechanistic studies. The reactions carried out and the corresponding conditions applied are summarized in Table 1. 
Both oxalic acid (dihydrate) and paraformaldehyde are required to cause $N$-methylation of the amines, only one single component shows no effect. Paraformaldehyde proved to be inert towards the amine under the applied reaction conditions. However, upon prolonged reaction times a

Table 1. Products and Reaction Conditions

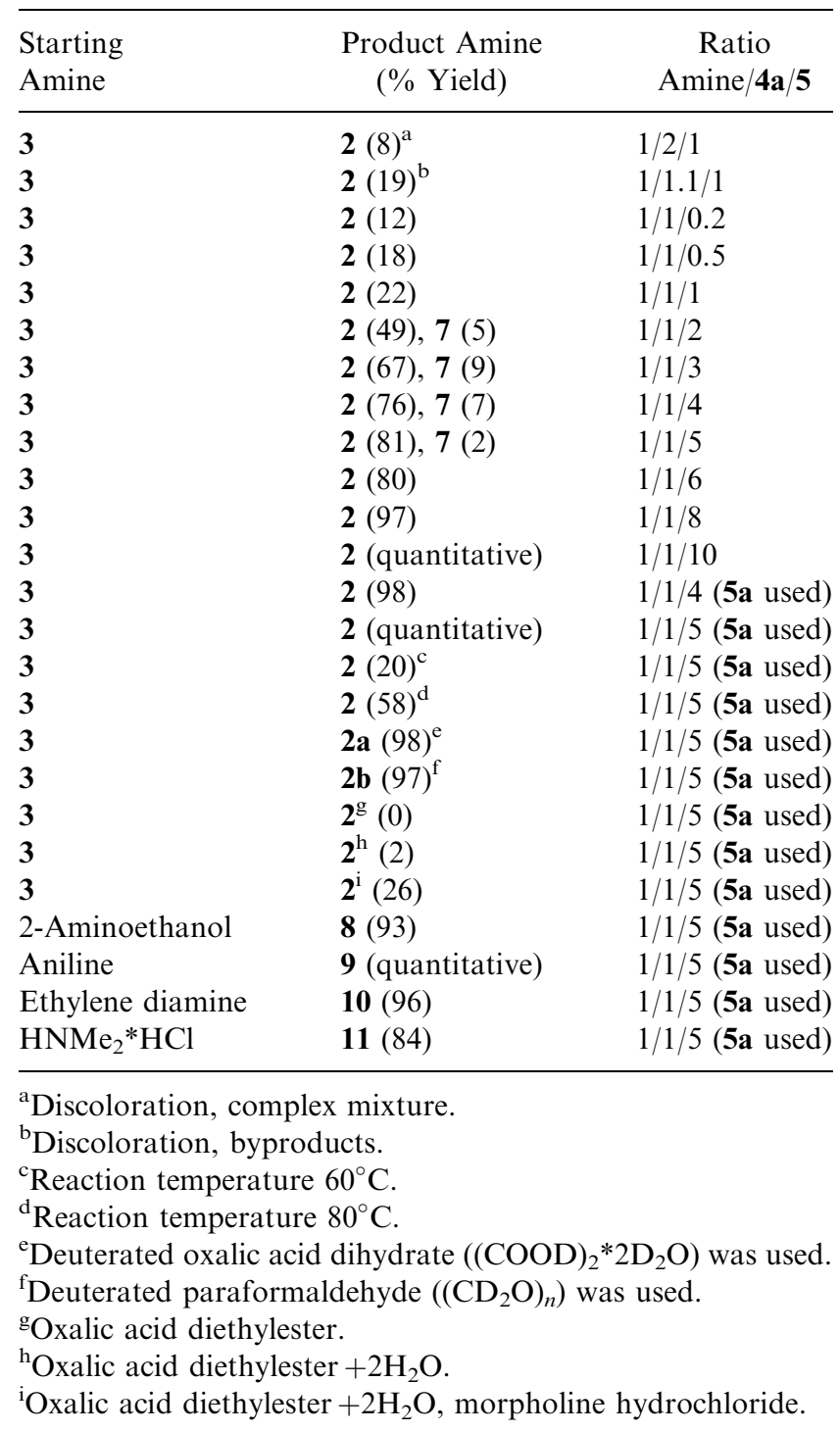


gradually increasing formation of chromophoric condensation products was observed. Oxalic acid and amine produced the corresponding oxalate without further reactions.

By addition of $\alpha$-tocopherol or $\gamma$-tocopherol, respectively, to the reaction mixture it was shown that the formation of the $N$-methylated products does not proceed according to a free radical process. Typical dimeric coupling products of the tocopherols, which are a sensitive indicator of homolytic processes, ${ }^{9,10}$ were not observed. The absence of those coupling products in the reaction mixture allowed to reliably rule out the occurrence of homolytic reactions during the $N$-methylation.

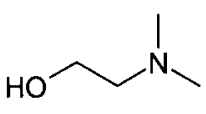

8<smiles>CN(C)c1ccccc1</smiles>

9

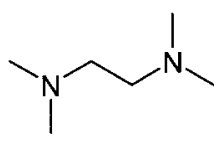

10

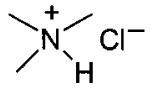

11

To establish the reaction mechanism, cross deuteration experiments were employed. With deuterated oxalic acid dihydrate (5a-D) and paraformaldehyde (4a), neat $N$-(monodeuteromethyl)morpholine (2a) was obtained. Using perdeuterated paraformaldehyde (4a-D) and 5a, isotopically pure $N$-(dideuteromethyl)morpholine (2b) was produced, a 1/1 mixture of 4a and 4a-D together with 5a afforded a $1 / 1$ mixture of $\mathbf{2}$ and $\mathbf{2 b}$. Interestingly, the system oxalic acid dihydrate/paraformaldehyde does not cause proton exchange at the methylene positions of $\mathrm{HCHO}$, and can thus be used for selective monodeutero-, bisdeutero-, or trideuteromethylation. This constitutes an interesting synthetic application of the novel reaction system, since in the system formic acid/formalin, conventionally used in Eschweiler-Clarke procedures, the isotopic integrity of HCHO is impaired, so that selective monodeuteromethylation or bisdeuteromethylation with this system was hitherto difficult.

The labeling experiments demonstrated that the $N$-methylation reaction proceeds via transfer of hydrogen to an intermediate which is formed by the reaction between amine and formaldehyde, probably the $N$-(hydroxymethyl)amine which is subsequently dehydrated in a protoncatalyzed reaction to the corresponding $N$-(methylene)iminium derivative (carbonium-iminium ion). The hydrogen transfer to this intermediate occurs dieither directly as hydride, or in a sequence of a proton and two electrons. 
With diethyl oxalate being used instead of oxalic acid dihydrate, no reaction was observed. The same result was obtained with a mixture of diethyl oxalate and two equivalents of water. This outcome proves the role of oxalic acid dihydrate in the reaction system as both acidic catalyst and hydrogen donor. With diethyl oxalate instead of $\mathbf{5 a}$ and amine hydrochloride instead of the free base, the methylated product was produced at rather low yield, indicating that the ester had been partly hydrolyzed to oxalic acid which acted as the reductant in turn.

\section{EXPERIMENTAL}

${ }^{1} \mathrm{HNMR}$ spectra were recorded at $300 \mathrm{MHz},{ }^{13} \mathrm{CNMR}$ spectra at $75.47 \mathrm{MHz}$, with $\mathrm{CDCl}_{3}$ as the solvent and TMS as the internal standard for amines, and $\mathrm{D}_{2} \mathrm{O}$ as the solvent with DSS as the internal standard for aminium hydrochlorides. ${ }^{13} \mathrm{C}$ peaks were assigned by means of HMQC and HMBC spectra. Chemical shift values are given in ppm. Chemicals were obtained from commercial sources or synthesized according to standard procedures. Even though the $N$-methylamines obtained are standard substances, their NMR data are listed in the following since literature values ${ }^{11,12}$ are inconsistent and difficult to compare.

\section{General Procedure for the Methylation of Primary or Secondary Amines}

A flask was charged with primary amine $(10 \mathrm{mmol})$, paraformaldehyde $(\mathbf{4 a}, 20 \mathrm{mmol})$ and oxalic acid dihydrate $(\mathbf{5 a}, 0.1 \mathrm{~mol})$ and briefly flushed with nitrogen. In the case of secondary amines, $10 \mathrm{mmol}$ of $\mathbf{4 a}$ and $50 \mathrm{mmol}$ of $\mathbf{5 a}$ were used. For amines with multiple amino functions, $10 \mathrm{mmol}$ of $\mathbf{4 a}$ and $50 \mathrm{mmol}$ of $\mathbf{5 a}$ were applied per methyl group to be introduced. The vessel was closed and heated to $100^{\circ} \mathrm{C}$ for $1 \mathrm{~h}$, and to $120^{\circ} \mathrm{C}$ for $10 \mathrm{~min}$. The reaction mixture was cooled to room temperature. The white, crystalline mass obtained was crushed, and calcium oxide $(0.1 \mathrm{~mol})$ suspended in $50 \mathrm{ml}$ of ethanol was added. The mixture was stirred vigorously for $30 \mathrm{~min}$, solids were removed by filtration, and the solvent was removed in vacuo to produce the pure amine.

For volatile amines, the crystalline reaction product was crushed and dissolved in water $(100 \mathrm{ml})$. The solution was adjusted to a $\mathrm{pH}$ of 10 by $10 \%$ aqueous sodium hydroxide and twice extracted with ethyl acetate $(10 \mathrm{ml})$. The combined organic phases were dried over $\mathrm{Na}_{2} \mathrm{SO}_{4}$, the desiccant was removed, and a $2 \mathrm{M}$ solution of hydrogen chloride in ethyl ether $(8 \mathrm{ml})$ was 
added. The resulting precipitate, the corresponding aminium hydrochloride, was separated by filtration, washed with ethyl acetate $(5 \mathrm{ml})$ and liberated from residual solvent in vacuo.

$N$-Methylmorpholine (2). $\mathrm{M}=101.14 \mathrm{~g} / \mathrm{mol}$, yield: $1.02 \mathrm{~g}(100 \%)$. ${ }^{1} \mathrm{H}$ NMR: $\delta 2.27$ (s, 3H, $\left.N-\mathrm{CH}_{3}\right), 2.39$ (t, 4H, $\left.N-\mathrm{CH}_{2}\right), 3.70$ (t, 4H, $\left.O-\mathrm{CH}_{2}\right)$. $N$-(Monodeuteromethyl)morpholine (2a). $\quad \overline{\mathrm{M}}=102.15 \mathrm{~g} / \mathrm{mol}$, yield: $1.00 \mathrm{~g}(98 \%) .{ }^{1} \mathrm{H}$ NMR $\left(\mathrm{D}_{2} \mathrm{O}\right): \delta 2.90\left(\mathrm{t}, 1 \mathrm{H}, N-\mathrm{CDH}_{2}\right), 3.21(\mathrm{dt}, 2 \mathrm{H}), 3.49$ $(\mathrm{dd}, 2 \mathrm{H}), 3.82(\mathrm{dt}, 2 \mathrm{H}), 4.12(\mathrm{dd}, 2 \mathrm{H}) .{ }^{13} \mathrm{CNMR}: \delta 43.6\left(\mathrm{t}, \mathrm{N}-\underline{\mathrm{CDH}_{2}}\right.$, $J=21.9 \mathrm{~Hz}), 53.6\left(\mathrm{~N}-\mathrm{CH}_{2}\right), 64.3\left(\mathrm{O}-\mathrm{CH}_{2}\right)$.

$N$-(Bisdeuteromethyl)morpholine (2b). $\mathrm{M}=103.16 \mathrm{~g} / \mathrm{mol}$, yield: $1.00 \mathrm{~g}$ $(97 \%) .{ }^{1} \mathrm{H} N M R\left(\mathrm{D}_{2} \mathrm{O}\right): \delta 2.92\left(\mathrm{t}, 1 \mathrm{H}, N-\mathrm{CD}_{2} \mathrm{H}\right), 3.20(\mathrm{dt}, 2 \mathrm{H}), 3.49$ (dd, $2 \mathrm{H}), 3.80(\mathrm{dt}, 2 \mathrm{H}), 4.12(\mathrm{dd}, 2 \mathrm{H}) .{ }^{13} \mathrm{CNMR}: \delta 43.6$ (pentett, $N-\mathrm{CD}_{2} \mathrm{H}$, $J=21.9 \mathrm{~Hz}), 54.0\left(\mathrm{~N}-\mathrm{CH}_{2}\right), 64.7\left(\mathrm{O}-\mathrm{CH}_{2}\right)$.

2-(Dimethylamino)-ethanol (8). $\mathrm{M}=89.13 \mathrm{~g} / \mathrm{mol}$, yield: $0.83 \mathrm{~g}(93 \%)$. ${ }^{1} \mathrm{H}$ NMR: $\delta 2.27\left(\mathrm{~s}, 6 \mathrm{H}, N-\left(\mathrm{CH}_{3}\right)_{2}\right), 2.46\left(\mathrm{t}, 2 \mathrm{H}, J=5.4 \mathrm{~Hz}, N-\mathrm{CH}_{2}\right), 3.61(\mathrm{t}$, $\left.2 \mathrm{H}, J=5.4 \mathrm{~Hz}, O-\mathrm{CH}_{2}\right) .{ }^{13} \mathrm{C} \mathrm{NMR}: \delta 45.6\left(N-\left(\underline{\mathrm{CH}}_{3}\right)_{2}\right), 58.5\left(N-\underline{\mathrm{C}}_{2}\right), 61.2$ $\left(\mathrm{O}-\mathrm{CH}_{2}\right)$.

$N, N$-Dimethylaniline (9). $\mathrm{M}=121.18 \mathrm{~g} / \mathrm{mol}$, yield: $1.22 \mathrm{~g}(100 \%)$. ${ }^{1} \mathrm{H} N M R: \delta 2.93\left(\mathrm{~s}, 6 \mathrm{H}, N-\left(\mathrm{CH}_{3}\right)_{2}\right), 6.63\left(\mathrm{~m}, 3 \mathrm{H},{ }^{\mathrm{Ar}} \mathrm{C} \mathrm{H}\right), 7.24(\mathrm{~m}, 2 \mathrm{H}$, $\left.{ }^{\mathrm{Ar}} \mathrm{CH}\right) .{ }^{13} \mathrm{C} \mathrm{NMR}: \delta 41.0\left(N-\mathrm{CH}_{3}\right), 113.1\left(\mathrm{C}^{2}, \mathrm{C}^{6}\right), 117.1\left(\mathrm{C}^{4}\right), 129.5\left(\mathrm{C}^{3}\right.$, $\left.\mathrm{C}^{5}\right), 151.1\left(\mathrm{C}^{1}\right)$.

$N, N, N^{\prime}, N^{\prime}$-Tetramethylethylene diamine (10). $\mathrm{M}=116.20 \mathrm{~g} / \mathrm{mol}$, yield: $1.11 \mathrm{~g}(96 \%) .{ }^{1} \mathrm{H}$ NMR: $\delta 2.18\left(\mathrm{~s}, 12 \mathrm{H}, N-\left(\mathrm{C}_{3}\right)_{2}\right), 2.36$ (s, 4H, $\left.N-\underline{C}_{2}\right)$. ${ }^{13} \mathrm{C}$ NMR: $\delta 41.2\left(\mathrm{~N}-\left(\mathrm{CH}_{3}\right)_{2}\right), 57.7\left(\mathrm{~N}-\mathrm{CH}_{2}\right)$.

Trimethylamine hydrochloride (11). $\mathrm{M}=95.57 \mathrm{~g} / \mathrm{mol}$, yield: $0.80 \mathrm{~g}$ (84\%). ${ }^{1} \mathrm{H}$ NMR $\left(\mathrm{D}_{2} \mathrm{O}\right): \delta 2.88\left(\mathrm{~s}, 9 \mathrm{H}, \mathrm{N}-\left(\mathrm{CH}_{3}\right)_{3}\right) .{ }^{13} \mathrm{C}$ NMR: $\delta 42.4$.

\section{CONCLUSION}

A mixture of paraformaldehyde and oxalic acid dihydrate in the molar ratio $1 / 5$ can be used for neat $N$-methylation of primary or secondary amines, paraformaldehyde and amine must be employed in equimolar ratios. As the reaction proceeds in the molten state, reaction temperatures of $100-120^{\circ} \mathrm{C}$ must be applied. No solvents are required, the toxic formalin and concentrated formic acid, as used in the conventional Eschweiler-Clarke methylation, are avoided. The methylated amines are obtained in excellent to quantitative yields, no quaternization of the amines is observed. Due to the elevated reaction temperatures necessary and the acidity of the reaction medium, the procedure is not apt for thermally labile or acid-sensitive amines. With either deuterated 
paraformaldehyde or deuterated oxalic acid a selective $N$-bisdeuteromethylation, respectively $N$-monodeuteromethylation, of amines is possible which could not be accomplished by the traditional Eschweiler-Clarke procedures.

\section{ACKNOWLEDGMENT}

The authors are grateful to the Christian-Doppler-Forschungsgesellschaft, Vienna, Austria, and to Lenzing AG, Lenzing, Austria, for financial support.

\section{REFERENCES}

1. Eschweiler, W. Ersatz von an Stickstoff gebundenen Wasserstoffatomen durch die Methylgruppe mit Hülfe von Formaldehyd. Chem. Ber. 1905, 38, 880-892.

2. Clarke, H.T.; Gillespie, H.B.; Weisshaus, S.Z. The action of formaldehyde on amines and amino acids. J. Am. Chem. Soc. 1933, 55, 4571-4587.

3. Leuckardt, R. Ueber eine neue Bildungsweise von Tribenzylamin. Ber. Dtsch. Chem. Ges. 1885, 18, 2341-2344.

4. Lukasiewicz, A. Reduction of Schiff bases by formic acid. Tetrahedron 1963, 19, 1789-1799.

5. Rosenau, T.; Potthast, A.; Kosma, P.; Chen, C.L.; Gratzl, J.S. Autocatalytic decomposition of $N$-methylmorpholine- $N$-oxide induced by Mannich intermediates. J. Org. Chem. 1999, 64, 2166-2167.

6. Holleman, A.E.; Wiberg, N. XIII. Die Gruppe der Halogene. In Lehrbuch der anorganischen Chemie; 91-100 Ed., De Gruyter: Berlin, New York, 1985, pp. 439, 657.

7. Lapidus, G.; Barton, D.; Yankwich, P.E. J. Kinetics and stoichiometry of the gas-phase decomposition of oxalic acid. Phys. Chem. 1964, 68, 1863-1865. Lapidus, G.; Barton, D.; Yankwich, P.E.J. Reversing hydrogen isotope effects on the rate of the gas-phase decomposition of oxalic acid. Phys. Chem. 1966, 70, 407-411. Lapidus, G.; Barton, D.; Yankwich, P.E.J. Intramolecular kinetic carbon isotope effect in the gas-phase decomposition of deuteriooxalic acid. Phys. Chem. 1966, 70, 1575-1578. Lapidus, G.; Barton, D.; Yankwich, P.E.J. Reversing intramolecular kinetic carbon isotope effect in the gas-phase decomposition of oxalic acid. Phys. Chem. 1966, 70, 3135-3139. 
8. Higgins, J.; Zhou, X.; Liu, R.; Huang, T.T.S.J. Theoretical study of thermal decomposition mechanism of oxalic acid. Phys. Chem. A 1997, 101, 2702-2708.

9. Machlin, L.J. Vitamin E: A Comprehensive Treatise, Marcel Dekker Inc.: New York, 1980.

10. Niki, E.; Matsuo, M. Peroxyl radical trapping reactions of $\alpha$-tocopherol in biomimetic systems. In Vitamin $E$ in Health and Diseases; Packer, L.; Fuchs, J., Eds.; Marcel Dekker Inc.: New York, 1993.

11. Pouchert, C.J.; Behnke, J. The Aldrich Library of ${ }^{13} C$ and ${ }^{1} H$ FT NMR Spectra; 1st Ed.; Aldrich Chemical Company Inc., 1993, 1.597A, $1.597 \mathrm{C}, 1.1250 \mathrm{C}$.

12. Kalinowski, H.O.; Berger, S.; Braun, S. ${ }^{13} C$-NMR-Spektroskopie, Thieme: Stuttgart, New York, 1984, 318.

Received in the USA March 19, 2001 